\title{
A SHORT PROOF OF A THEOREM OF PLANS ON THE HOMOLOGY OF THE BRANCHED CYCLIC COVERINGS OF A KNOT
}

\author{
BY C. MCA. GORDON
}

\section{Communicated by N. E. Steenrod, July 6, 1970}

Let $K \subset S^{3}$ be a (tame) knot, with complement $C=S^{3}-K$, and let $\tilde{C}$ be the infinite cyclic covering of $K$, i.e. the covering of $C$ corresponding to the commutator subgroup of $\pi_{1}(C)$. The group of covering translations of $\tilde{C}$ is $H_{1}(C)$, which is infinite cyclic by Alexander duality; this gives an action of $\boldsymbol{Z}$ on $H_{1}(\tilde{C})$, and so $H_{1}(\tilde{C})$ becomes a $\Lambda$ module, where $\Lambda$ is the integral group ring of $\boldsymbol{Z}$. We identify $\Lambda$ with the ring of polynomials in a single variable $t$, (positive and negative powers of $t$ being allowed), with integral coefficients. (See [4].)

The $k$-fold branched cyclic covering of $K, M_{k}(k \geqq 1)$ is defined by taking the covering of $C$ corresponding to the kernel of the composition:

$$
\pi_{1}(C) \rightarrow H_{1}(C) \cong Z \rightarrow Z_{k},
$$

and branching about $K$. (For more details, see [1], [4].) $M_{k}$ is a closed, orientable 3-manifold: for example, $M_{1}$ is just $S^{3}$.

If $M(t)=\left(m_{i j}(t)\right), m_{i j}(t) \in \Lambda$, is a presentation matrix for $H_{1}(\tilde{C})$ as a $\Lambda$-module, then it can be shown that a presentation matrix for $H_{1}\left(M_{k}\right)$ $(k>1)$ as an abelian group is obtained by substituting for each entry $m_{i j}(t)$, which is some finite formal sum, $\sum_{\nu} \alpha_{\nu} t^{\nu}$, say, the $k \times k$ block $\sum_{\nu} \alpha_{\nu} T_{k}^{\nu}$, where the summation indicates ordinary matrix addition, and $T_{k}$ is the $k \times k$ matrix:

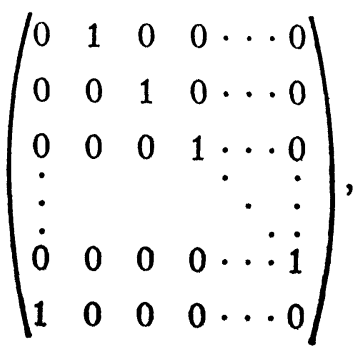

AMS 1970 subject classifications. Primary 55A10, 55A25.

Key words and phrases. Classical knot theory, branched cyclic covering, Seifert matrix, Plans' Theorem, direct double. 
(and $T_{k}^{0}$ is defined to be the $k \times k$ identity matrix). (See [2], [4].) Call the matrix obtained from $M(t)$ in this way $M\left(T_{k}\right)$.

Now a geometrical description of $\tilde{C}$, in terms of an orientable surface spanning $K$, shows that we may take $M(t)$ to be of the form $t V-V^{T}$, where $V$ is a $2 h \times 2 h$ matrix over $\boldsymbol{Z}(h \geqq$ the genus of $K)$ and $V^{T}$ is the transpose of $V$. (See [1], [6].) $M\left(T_{k}\right)$ is then $2 h k \times 2 h k$, but Seifert showed (see [1], [6]) that it is in fact equivalent (in the sense of presenting the same abelian group) to a $2 h \times 2 h$ matrix, $F_{k}$ say. In [5], Plans shows that this $F_{k}$ can be expressed in terms of two matrices $P_{k}$ and $Q_{k}$, which in turn are defined by certain recurrence relations analogous to those defining the Fibonacci numbers. These facts are used to effect a diagonalisation of $F_{k}$, from which some interesting general conclusions about $H_{1}\left(M_{k}\right)$ are drawn, perhaps the most striking being the following:

Theorem (Plans). If $k$ is odd, then $H_{1}\left(M_{k}\right)$ is a direct double, i.e. $H_{1}\left(M_{k}\right) \cong G \oplus G$, for some $G$.

The proof given in [5] is rather long and involved, and it is the purpose of this note to show that, although $F_{k}$ is smaller than $M\left(T_{k}\right)$, the above result actually follows very easily from an examination of the big matrix.

Proof. $M(t)=t V-V^{T}$. So by a suitable sequence of row interchanges and column interchanges, $M\left(T_{k}\right)$ can be brought into the form:

$$
\left(\begin{array}{ccccccc}
-V^{T} & V & 0 & 0 & \cdots & 0 & 0 \\
0 & -V^{T} & V & 0 & \cdots & 0 & 0 \\
0 & 0 & -V^{T} & V & \cdots & 0 & 0 \\
\vdots & & & \cdot & \cdot & \vdots & \vdots \\
\vdots & & & & \cdot & \vdots & \vdots \\
\vdots & 0 & 0 & 0 & \cdots & -V^{T} & \dot{V} \\
V & 0 & 0 & 0 & \cdots & 0 & -V^{T}
\end{array}\right)
$$

a $k \times k$ matrix of $2 h \times 2 h$ blocks.

Now if $k$ is odd, $k=2 r+1$ say, a further sequence of row interchanges gives a matrix in which the rows of blocks occur in the order (numbering them according to their positions in the old matrix): $r+1, r+2, \cdots, 2 r+1,1,2, \cdots, r$. It is easy to see that this new matrix is skew-symmetric. We illustrate the case $k=7$ : 


$$
\left(\begin{array}{ccccccc}
0 & 0 & 0 & -V^{T} & V & 0 & 0 \\
0 & 0 & 0 & 0 & -V^{T} & V & 0 \\
0 & 0 & 0 & 0 & 0 & -V^{T} & V \\
V & 0 & 0 & 0 & 0 & 0 & -V^{T} \\
-V^{T} & V & 0 & 0 & 0 & 0 & 0 \\
0 & -V^{T} & V & 0 & 0 & 0 & 0 \\
0 & 0 & -V^{T} & V & 0 & 0 & 0
\end{array}\right) .
$$

But it is well known (see for example [3, p. 52]) that any skewsymmetric $2 n \times 2 n$ matrix over $\boldsymbol{Z}$ is equivalent to a block diagonal matrix of the form:

$$
\sum_{i=1}^{n}\left(\begin{array}{cc}
a_{i} & 0 \\
0 & a_{i}
\end{array}\right) \quad\left(a_{i} \geqq 0\right),
$$

and hence presents a direct double.

\section{REFERENCES}

1. R. H. Fox, A quick trip through knot theory, Topology of 3-Manifolds and Related Topics (Proc. Univ. of Georgia Inst., 1961), Prentice-Hall, Englewood Cliffs, N. J., 1962, pp. 120-167. MR 25 \#3522.

2. L. Goeritz, Die Betti'schen Zahlen der zyclischen Überlagerungsräume der Knotenaussenräume, Amer. J. Math. 56 (1934), 194-198.

3. C. C. MacDuffee, The theory of matrices, Springer, Berlin, 1933; reprint, Chelsea, New York, 1946.

4. L. P. Neuwirth, Knot groups, Princeton Univ. Press, Princeton, N. J., 1965.

5. A. Plans, Contribution to the study of the homology groups of the cyclic ramified coverings corresponding to a knot, Rev. Acad. Ci. Madrid 47 (1953), 161-193. (Spanish) MR 15, 147.

6. H. Seifert, Über das Geschlect von Knoten, Math. Ann. 110 (1934), 571-592.

Florida State University, Tallahassee, Florida 32306 